\title{
DESCLASADOS Y DESIGUALES: MODELO EXPLICATIVO RELACIONAL DE MOVILIDAD DE CLASES SOCIALES
}

\author{
DESCLASSIFICADOS E DESIGUAIS: MODELO EXPLICATIVO \\ RELACIONAL DE MOBILIDADE DE CLASSES SOCIAIS
}

\author{
DOWN-CLASSES AND UNEQUALS: RELATIONAL EXPLANATORY \\ MODEL OF SOCIAL CLASS MOBILITY
}

DOI: $10.22481 /$ rbba.v10i02.9677

Diana Esperanza Oliveros Fortiche

Bogotá, Distrito Especial, Colombia

ORCID: https://orcid.org/0000-0001-8817-8361

Dirección electrónica: doliverosf@ucentral.edu.co

\section{RESUMEN}

Este artículo de investigación doctoral presenta un modelo explicativo relacional entre el sistema capitalista, la sociedad de consumo y las estrategias de movilidad de clases sociales medias y bajas en Colombia. Su objetivo es sustentar la hipótesis que las prácticas de consumo desarrolladas durante los procesos de ingreso y acomodación en clases medias reproducen y naturalizan la desigualdad. En su base teórica confluyen categorías interdisciplinarias de las ciencias sociales como antropología, sociología, economía y psicología. Su metodología de corte mixto utiliza técnicas cualitativas de entrevista biográfica y cuantitativas de encuesta. Entre los principales resultados emerge la paradoja: el plan de escape de la inequidad reproduce una nueva inequidad legitimada por los agentes de clase a través de estrategias de acumulación de ciertos capitales, de prácticas de consumo y de la conformidad con los mecanismos constitutivos de la desigualdad. 
Palabras clave: Clases Medias. Movilidad Social. Consumo. Desigualdad Social.

\title{
RESUMO
}

Este artigo de pesquisa de doutorado apresenta um modelo explicativo relacional entre o sistema capitalista, a sociedade de consumo e as estratégias de mobilidade das classes sociais média e baixa na Colômbia. Seu objetivo é sustentar a hipótese de que as práticas de consumo desenvolvidas durante os processos de renda e acomodação das classes médias reproduzem e naturalizam a desigualdade. Sua base teórica converge categorias interdisciplinares das ciências sociais como antropologia, sociologia, economia e psicologia. Sua metodologia mista usa entrevista biográfica qualitativa e técnicas de pesquisa quantitativa. Entre os principais resultados, surge o paradoxo: o plano de fuga à desigualdade reproduz uma nova desigualdade legitimada pelos agentes de classe por meio de estratégias de acumulação de certos capitais, práticas de consumo e cumprimento dos mecanismos constitutivos da desigualdade.

Palavras-chave: Classes médias. Mobilidade social. Consumo. Desigualdade social.

\begin{abstract}
This doctoral research article presents a relational explanatory model between the capitalist system, the consumer society and the mobility strategies of the middle and lower social classes in Colombia. Its objective is to support the hypothesis that the consumption practices developed during the processes of income and accommodation in the middle classes reproduce and naturalize inequality. Its theoretical base converges interdisciplinary categories of social sciences such as anthropology, sociology, economics and psychology. Its mixed-cut methodology uses qualitative biographical interview and quantitative survey techniques. Among the main results, the paradox emerges: the plan to escape inequality reproduces a new inequity legitimized by class agents through strategies of accumulation of certain capitals, consumption practices and compliance with the constitutive mechanisms of inequality.
\end{abstract}


Keywords: Middle Classes. Social Mobility. Consumption. Social inequality.

\section{INTRODUCCIÓN}

Colombia se encuentra entre los países más desiguales de Latinoamérica. Reducir esta desigualdad histórica, junto con la finalización del conflicto interno, son objetivos presentes en cada uno de los planes de gobierno presidenciales desde el siglo pasado, y aunque se ha disminuido los índices de desigualdad, el país sigue ocupando los tres primeros puestos con respecto al índice de desigualdad Gini, según cifras de la CEPAL y el Banco Mundial para el 2019.

Una de las formas de identificar la reducción de la desigualdad es el aumento porcentual de las clases medias frente a los índices de clase baja y pobreza, puesto que las clases medias son vistas como base del desarrollo económico y democrático de un país. En 2019, el actual gobierno de Iván Duque Márquez (2018-2022) daba una excelente noticia para Colombia, nos habíamos convertido en un país de clase media, a través de valorar el ingreso total de los hogares y permitir que personas que se encontraban en clase baja con ingresos mínimos fueran reconocidos de la noche a la mañana como clase media (VIVAS PACHECO, 2019). ¿Cómo sucedió? Sencillamente se acogió los lineamientos del Banco Mundial que determina una clase media y una media vulnerable a partir de los ingresos diarios. Así, las personas de clase media establecida son los que perciben ingresos diarios entre 10 y 50 USD y los de media vulnerable son los que reciben entre 4 y 10 dólares diarios. Así las cosas, en un país de pobreza como Colombia, un grupo familiar que gane un poco más de 4 USD diarios (que equivale a la compra de quinientos gramos de carne) es clasificado como clase media vulnerable (KAIPPER CERATT, 2016). Evidentemente esta determinación "política" no le da una verdadera existencia a la clase media vulnerable, por el contrario invisibiliza la pobreza y pone a los agentes de clase baja de cara a las falencias de capitales y habitus de acumulación característicos de una real clase media.

Este fenómeno actual que atraviesan los promovidos a clase media a partir de paupérrimos ingresos, no se distancia de lo que históricamente han sido los procesos de enclasamiento y de desigualdad vividos por personas pertenecientes a clases bajas colombianas. La desigualdad y el ingreso a una clase superior son procesos que se desarrollan de manera

Revista RBBA $\mid$ Revista Binacional Brasil Argentina Vol. 10, num. 2, dez/2021, p. 78-92 
conjunta, pues como Deaton (2015) advierte, es imposible referirse al progreso material y estatutario sin abordar simultáneamente la historia de desigualdad que lo sustenta.

El progreso material está asociado a la desigualdad por múltiples razones. Una razón importante es la correlación entre los consumos de bienes posicionales o estatutarios y la existencia de una mayor desigualdad (GORGA, LEITES Y VIGORITO, 2016). El consumo de bienes posicionales es denominado estatutario, posicional o conspicuo porque tiene por objetivo el uso o apropiación de mercancías y posesiones para demostrar o mejorar estatus (CAMPBELL, 1995; SCHOR, 2015; AGO, 2015). Sobre los procesos de enclasamiento desarrollados bajo condiciones de inequidad se construyó la indagación doctoral que da origen al modelo explicativo relacional de movilidad de clases sociales, presentado a continuación.

\section{BASE TEÓRICA}

La construcción de la base teórica para la comprensión de las estrategias o procesos de movilidad social recurre a una mirada interdisciplinar que complejiza y amplia el análisis de los resultados hallados. Las principales categorías teóricas abordadas son: clase social, movilidad y consumo, enmarcados en sociedad de consumo y sistema capitalista.

Para Bourdieu (1990) la clase social es un "conjunto de agentes ocupando posiciones similares que, situados en condiciones similares y sometidos a condicionamientos similares, tienen todas las probabilidades de tener disposiciones e intereses similares, luego de producir prácticas similares y parecidas tomas de posición” (p. 30). Así, lo repetitivamente "similar" en las clases sociales está dado por el lugar que ocupan en el espacio social, los condicionamientos o habitus que practican y por la acumulación de capitales económicos, sociales, culturales y simbólicos que poseen. Sumado a estos capitales se encuentra un quinto tipo de capital que afecta el espacio que ocupa la clase en un espacio social, este es el Capital Tecnológico de Información y Comunicación, TIC (OLIVEROS, 2017).

El capital TIC es una nueva especie de capital similar al cultural que se mide en sus propios términos y habitus. Sus formas son: incorporado, objetivado e institucionalizado. El capital TIC incorporado es el aprendido en el hogar o grupo social de origen. El capital TIC objetivado es representado en bienes que se identifican por criterios técnicos y los significados y usos que sobre ellos se producen. El capital TIC institucionalizado es representado por aprendizajes formales, certificados o títulos que dan cuenta de un saber especializado y técnico.

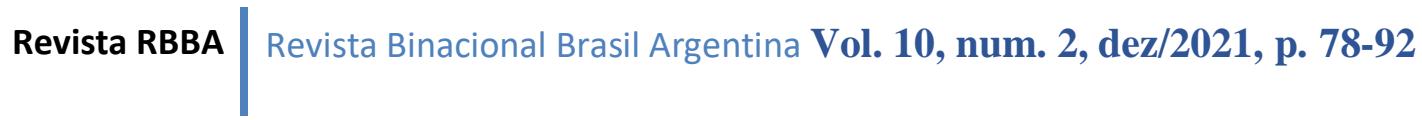


El capital TIC como los demás capitales están sujetos a un poder constructor o de imposición de la visión legítima del mundo, ya que están definidos por el valor que es socialmente reconocido o legitimado y, por tanto responden a clasificaciones y representaciones existentes en una sociedad particular sobre el género, la edad, el estatus, entre otros (OLIVEROS, 2017). Las prácticas de apropiación o acumulación de los capitales se logran a través del habitus de los agentes, es así como los habitus están determinados por las normas y reglas de clase. Los habitus de los agentes también son condicionados por sus estilos de vida, experiencias y prácticas de clase. Los habitus de clase se asumen como algo dado por sentado e imponen lo que puede o no permitirse, marca límites y diferencias confiriendo, al fin y al cabo, un sentido de realidad al indivíduo.

La movilidad social, en este contexto de habitus de clase, es vista desde los enclasamientos y desclasamientos que realizan los sujetos de clases sociales en donde fijan diferencias, producto de sus condiciones estructurales (objetivas), pero también de sus prácticas (subjetivas o incorporadas) para mantener o disolver estas distinciones. Así, las distinciones son "un movimiento significativo en la posición económica, social y política del indivíduo" (MILLER, 1995, p. 26). Dicho movimiento puede ser ascendente, descendente u horizontal por los diferentes estratos en una estructura social.

Toda movilidad social requiere de aprendizajes y de transformaciones identitarias, acomodamientos y cambios que se visibilizan en la identidad individual, dado que "las identidades están para vestirlas y mostrarlas, no para quedarse con ellas y guardarlas" (BAUMAN, 2007, p. 188), en este sentido los juicios de clasificación y estratificación en principio son criterios de diferencia, pero también de desigualdad social, donde: no hay diferencia socialmente conocida y reconocida más que por un sujeto capaz, no solamente de percibir las diferencias, sino de reconocerlas como significantes (BOURDIEU, 1990).

El habitus de enclasamiento se desarrollan dentro de una cultura del consumo que marca un desplazamiento de la mercancía hacia el centro de la vida cotidiana. Desde esta perspectiva, la publicidad, los medios de comunicación masivos y el marketing son los vehículos que conducen a una búsqueda constante de nuevas modas, estilos, sensaciones y experiencias. En esta cultura del consumo, el consumidor vive dentro de un mundo de bienes y se comporta de manera irracional debido al fetichismo que mantiene con las mercancías. El consumo proporciona símbolos estatutarios que ayudan a definir, reforzar y comunicar las identidades que poseen los consumidores (BHATTACHARJEE, BERGER y MENON, 2014).

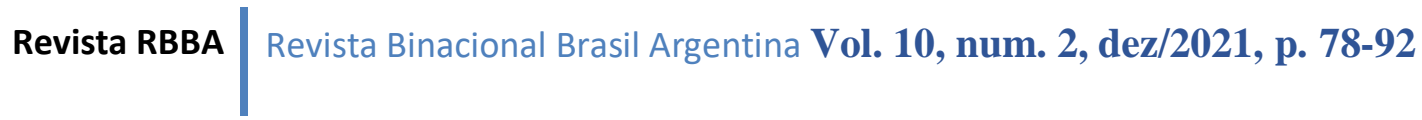


Los símbolos estatutarios están contenidos en los objetos. El signo o significado atribuido al objeto es producto de lo cultural y de lo social, por ello el significado es variable y dependiente del espacio social en el que se circulan dichos objetos y en donde se encuentra el consumidor. El consumidor produce un objeto en la medida en que lo vincula a su cotidianidad y experiencia de vida. Entre esos objetos, los relacionados con su identidad y vida emocional y la disputa por su posición, se determinarán como bienes estatutarios pues están cargados de valor simbólico suficiente para identificar una identidad grupal y con ello una posición de clase social (GORGA, LEITES y VIGORITO, 2016). Tanto el consumo de bienes estatutarios como el capitalismo se integran al sistema social "en el que predominan de forma hegemónica los procesos de circulación y acumulación del capital a la hora de proporcionar y configurar las bases materiales, sociales e intelectuales para la vida en común" (HARVEY, 2014, p.22).

Desde una mirada de medios y modos de producción, la sociedad de consumo está en relación intrínseca con el capitalismo como sistema económico, social y de producción dominante. El capitalismo es un sistema que favorece la propiedad privada sobre los medios de producción y la búsqueda de la maximización del benefício en un mercado competitivo y libre respecto a la oferta y la demanda (LEWELLEN, 2002). Estas características del capitalismo pueden llegar a ser naturalizadas, puesto que, una vez que el capitalismo y el neoliberalismo penetran el sentido común de las sociedades, son consideradas formas necesarias y naturales de regulación del orden social (HARVEY, 2007). El neoliberalismo, como ideología de la liberación del mercado de la regulación gubernamental, crea el espacio fértil para el desarrollo capitalista, por ello el neoliberalismo y el capitalismo se mantienen en una simbiosis difícil de disolver (LEWELLEN, 2002).

El capitalismo genera profundas contradicciones como la discriminación, las disparidades de renta y riqueza, y la intromisión de los procesos productivos y de consumo en la red de la vida social y biológica de los sujetos, convirtiéndose en un rasgo de la sociedad de consumo. Featherstone (2001) se centra en la cultura del consumo como determinante de la sociedad posmoderna o sociedad de consumo. El término de cultura del consumo surge en los años ochenta para indicar un desplazamiento de la mercancía hacia el centro de la vida cotidiana (PAYNE, 2002). Es así como cultura, consumo, sociedad y capitalismo tejen una intrincada relación que afecta directamente al sujeto de clase y a su movilidad.

Revista RBBA $\mid$ Revista Binacional Brasil Argentina Vol. 10, num. 2, dez/2021, p. 78-92 


\section{METODOLOGÍA}

El tipo de investigación fue de corte mixto, secuencial y derivativo (HERNÁNDEZ SAMPIERI, FERNÁNDEZ COLLADO y BAPTISTA, 2008). Tuvo dos fases: una cualitativa que contó con un diseño biográfico centrado en el método de historia de vida y en la técnica de entrevista a profundidad; la segunda fase fue cuantitativa, por ello la aclaración de derivativa, con un diseño no experimental, de tipo transversal correlacional.

Los sujetos en la fase cualitativa fueron 6 personas para las historias de vida y 5 más con entrevistas complementarias. En la fase cuantitativa el tamaño de muestra fue de 122 personas pertenecientes a clases medias y bajas, distribuidas así: 56 personas de clase baja, de ellas 29 mujeres y 27 hombres y 66 personas de clase media, 31 hombres y 35 mujeres.

Para los instrumentos de recolección de construyó un guion para entrevistas semiestructuradas y en cuestionario encuestal. Para el análisis de información se usó codificación abierta, axial con apoyo de Atlas.ti 7, y análisis de tipo factorial multivariado que incluyó análisis de componentes principales, correspondencias simples y múltiples, análisis lexicométricos de frecuencia de palabras, palabras en contexto y segmentos característicos.

\section{ESTRATEGIAS DE MOVILIDAD: ACUMULACIÓN DE CAPITALES Y PRÁCTICAS DE CONSUMO}

De Botton (2004) menciona que en la sociedad actual se posee la falsa idea que cualquiera puede obtener estatus sin importar su origen o condición. Y aunque los colombianos saben lo difícil de escalar socialmente, guardan la esperanza de la llegada de una situación mejor. La aspiración de ascender socialmente es transmitida por los padres, puesto que fijarse metas de ascenso social, aprender a lograrlo, así como las primeras oportunidades de estudio o trabajo es un legado de los padres, ya que son ellos los encargados de formar a los hijos con el ejemplo y abrirles las puertas a mejores relaciones y al conocimiento de un contexto menos violento a partir de cambiarlos de lugar de vivienda o de colegio.

Aprovechar la oportunidad dada por los padres es una de las primeras acciones para obtener en el futuro una mejor posición social. Becker (1993) propone un modelo simple de inversión de los padres en los hijos como prevención de la desigualdad y aseguramiento de la movilidad intergeneracional. Este tipo de inversión de los padres se expresa en herencias o

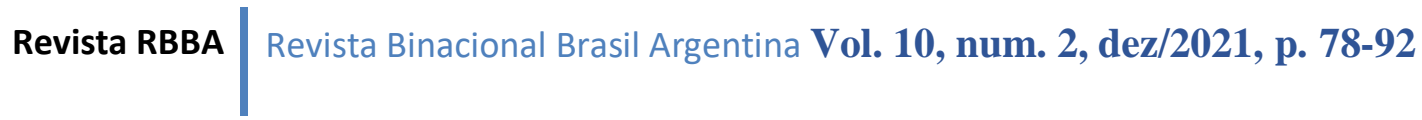


regalos, y de legados como la educación o la formación de capital humano. Becker hace un comparativo entre familias y encuentra que las familias ricas invierten tanto en el capital humano como no humano de sus hijos y que entre hijos se mantienen diferencias por las preferencias de los padres. Siendo este tipo de inversión fundamental para igualar las oportunidades de las generaciones posteriores. En la investigación se encontró que además de la inversión en educación (capital cultural) por los padres, se ubican otras estrategias de movilidad relacionadas con el trabajo, el esfuerzo, el ahorro, las conexiones (o capital social), la fe, fijarse metas y realizar sacrificios, vestirse y migrar.

En princípio, es la educación básica (primaria y secundaria) y la técnica las que abren el camino para una mejor ocupación o trabajo. La educación superior es un paso mayor para el bienestar, el ascenso y la cualificación laboral y, por lo tanto, es una estrategia más certera para la movilidad. El trabajo provee recursos y junto con la educación, forman la principal dupla estratégica para la movilidad. El trabajo, como la educación, se relacionan con la tercera estrategia, las conexiones o lograr capital social.

Cada una de las estrategias de educación, trabajo y conseguir conexiones implica esfuerzo y lucha, ya que cada persona que supera las difíciles condiciones estructurales en Colombia es definida como berraco(a), término que según la RAE es dado a una "persona que se desempeña muy bien", pero también a alguien "que logra con trampa o triquiñuelas obtener sus fines"; no cualquiera de estas dos formas de logro hace a un luchador digno de reconocimiento, ya que llegar lejos por contactos, esfuerzo y trabajo es la manera digna de hacerlo, mientras el camino indigno es aquel marcado por la trampa y el dinero fácil.

La berraquera no es lo mismo que el esfuerzo y sacrificio debido a que el esfuerzo por moverse socialmente, además de obedecer a los limitantes estructurales, está relacionado con la desconfianza en políticas e instituciones estatales y la triste certeza de que solo a través de las propias manos o el esfuerzo propio se transforman estas precarias condiciones, así el camino loable y ético es aquel marcado por el esfuerzo y el sacrificio.

Este camino de dificultad es alivianado por la creencia y la fe, comprendiéndose mejor porque Colombia es un país de fe, puesto que más del $70 \%$ de los colombianos se identifican como católicos. Aunque los procesos de secularización vividos en los últimos años han disminuido el número de católicos, se ha aumentado la migración de fieles hacia movimientos religiosos como el pentecostalismo. Paradójicamente la secularización, en lugar de debilitar la religión ha desencadenado un proceso constante de recomposición religiosa (BELTRÁN,2013),

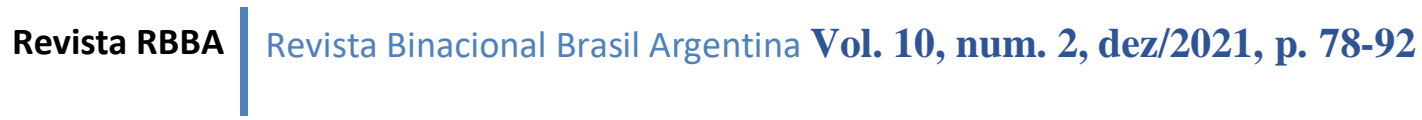


manteniendo la fe intacta en una religión, y con esto la esperanza en un dios que puede cambiar el curso de las cosas por más cruentas que se presenten.

La fe y las creencias dan algunas certezas sobre el éxito en las estrategias de movilidad y se convierte en la ciencia detrás de la creencia de "querer es poder", a la que Cabanas e Illouz (2007) señalan como ciencia de la felicidad por la cual se procura la autorrealización, el crecimiento personal y la mejora social, lo que legitima la idea que la riqueza, el éxito y la salud es fruto de los propios actos.

Esta ciencia, según los autores, podría ser parte de una filosofía neoliberal y del capitalismo de consumo. El éxito, de acuerdo a la ciencia de la felicidad se puede lograr, a partir de convencimientos (creencias, fe) y de manejar un pensamiento positivo con las metas trazadas. Lo cierto es que, para el caso colombiano, la fe, las creencias y el trazarse metas tienen su origen en una perfecta hibridación entre la religión, corrientes de búsqueda de la felicidad y la autorrealización originada en la experiencia de desigualdad y en la profunda desconfianza en la acción del Estado. Un Estado en construcción que aún no consigue atender necesidades de bienestar en cuanto a seguridad, educación o salud, pero que celebra tener una de las democracias más sólidas en Latinoamérica, democracia opacada por los índices de corrupción y la violencia (ARIAS, 2017).

Autogestionar la propia movilidad requiere, además de lo ya expuesto, prácticas sutiles que intervienen en la estética, como es el caso del vestirse. Pedraza (2011) señala que además de la estética, están el decoro y la corrección como parte de las consideraciones de la conducta social alrededor del vestir. La estética es dada por la concordancia entre estilos y colores del atuendo y se relaciona con el gusto, mientras el decoro refleja la moral y la corrección es la relación entre la vestimenta y la posición social de la persona. Y aunque en los relatos personales sobre la movilidad no se alude al decoro, sí se entrelazan la estética y la corrección como parte de la acción estratégica del saberse vestir.

El ahorro aparece como un gasto postergado y por ello es una acción estratégica relacionada con el consumo, en especial con el consumo de bienes estatutarios. El ahorro no es una práctica extendida en la cultura colombiana, y tal como afirma el estudio de la Banca de las Oportunidades, Federación de Aseguradores Colombianos y Superintendencia Financiera de Colombia (2018), solo un 18,5\% de los hogares tiene ahorros, de estos un poco más del 70\% los tiene en bancos y el restante los guarda en alcancía, debajo del colchón o en cadenas de

Revista RBBA $\mid$ Revista Binacional Brasil Argentina Vol. 10, num. 2, dez/2021, p. 78-92 
ahorros. Los ahorros son usados en principio para consumo, cubrir imprevistos o pagar la educación.

En relación específica con la acumulación de los capitales se encontró que el capital cultural puede perder relevancia al no estar en correspondencia con otros capitales como el económico, social, TIC y su respectiva transformación en capital simbólico. Perdiendo relevancia el capital cultural es el social y el TIC que lo reemplazan, por esta razón, se observó la posición privilegiada del capital social, y la alta frecuencia de relatos sobre la importancia del celular en los espacios sociales, culturales y laborales, convirtiéndolo en un bien de prestigio dependiendo de la marca y la calidad del aparato.

El capital simbólico forma parte de todos los capitales. Este capital es una forma de poder de reconocimiento. Se acumula capital simbólico cuando los elementos significativos que componen los otros capitales se resignifican y son convertidos en lo más valioso. Los objetos valiosos son más preciosos por las marcas que poseen. Además se encontró que este tipo de bien están vinculados a la construcción individual como la distinción, el respeto y el prestigio.

Distinguirse junto con el enclasarse son prácticas de reproducción social. Dichas prácticas están supeditadas a los habitus que siguen los agentes en un campo, determinado por las clases sociales y sus tensiones: "Lo que hacen para distinguirse de otras clases es estudiar, comportarse decentemente, tener bienes y capacidad adquisitiva" (mujer, 48 años, clase media). No seguir dichas prácticas de enclasamiento o distinción terminan por degradar socialmente al agente de clase que busca ascender, así que estas estrategias se incorporan y son definidas como legítimas.

\section{MODELO EXPLICATIVO RELACIONAL DE MOVILIDAD DE CLASES SOCIALES}

En la figura 1 se establece el modelo que presenta las categorías más significativas encontradas durante la investigación dadas desde las estrategias que realizan los agentes de clase para enclasarse y/o mantenerse en una clase social superior y no desclasarse a menos. Este modelo establece relaciones con la desigualdad a la vez que tiene en cuenta las influencias macrosociales de la cultura y la sociedad de consumo y el sistema capitalista. Su objetivo es brindar una lectura compleja y necesaria sobre las paradojas generadas en las relaciones del consumo con el ascenso estatutario y el enclasamiento.

Revista RBBA $\mid$ Revista Binacional Brasil Argentina Vol. 10, num. 2, dez/2021, p. 78-92 
En el modelo relacional se observa que las estrategias de movilidad están asociadas al campo de la desigualdad puesto que enclasarse y desclasarse lleva al sujeto a acciones para desigualarse del contexto que lo constriñe. La meta de la movilidad ascendente es la misma que la del enclasamiento, es decir, lograr pertenecer a una mejor clase social.

La movilidad descendente trae un desclasamiento que implica exclusión estatutaria. La movilidad ascendente y descendente se interrelaciona con momentos de enclasamiento y desclasamiento, la diferencia entre el desclasamiento para ascender socialmente con el desclasamiento dado por una pérdida de estatus es la valoración negativa de este último y el esfuerzo que el sujeto realiza para no perder el estatus logrado.

Enclasarse requiere del aprendizaje y manejo de las diferencias. Un agente enclasado aprende a desigualar a los otros para posicionarse y mantener el estatus, ya que para desarrollar esta tarea aprende sobre los aspectos de diferencia (desigualdad) que dominan el comportamiento e identidad de la clase a la que quiere pertenecer o integrarse.

En los procesos de movilidad se requiere del logro de habitus de acumulación de capitales y de prácticas de consumo estatutario y de desigualdad que coexisten y se relacionan entre ellas. Respecto a la acumulación de capitales: cultural, social, económico y TIC se establecen como principales aquellos que producen de manera más efectiva percepción de estatus como el TIC y el social. El capital económico se muestra esquivo y es solo gracias a la objetivación por medio de bienes que se hace presente. La consecución de estos bienes requiere de esfuerzo para su logro. Las prácticas de consumo estatutario se producen en relación con el campo del mercado y están matizadas por la exhibición que se hace sobre los bienes de valor estatutario. Los rituales de ostentación son los más importantes para el logro conspicuo de estatus.

Revista RBBA $\mid$ Revista Binacional Brasil Argentina Vol. 10, num. 2, dez/2021, p. 78-92 
Figura 1. Modelo explicativo relacional de movilidad de agentes de clase

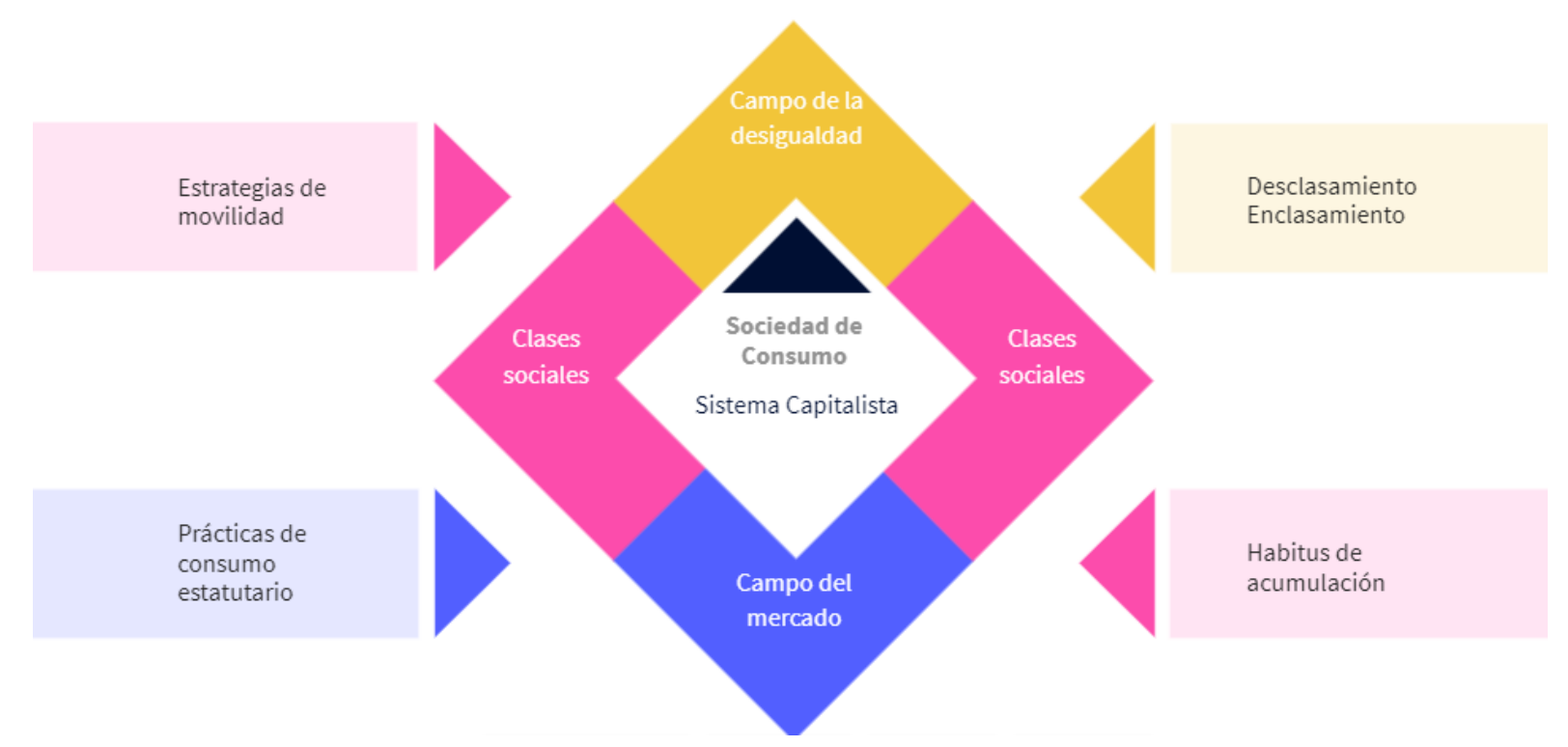

Fuente: Elaboración propia.

El campo de la desigualdad entra en relación con la acumulación de los capitales y las prácticas de consumo. Un aspecto preponderante de esta relación es su determinación a partir de su correspondencia con la sociedad de consumo y el mismo sistema capitalista. Es así como este Modelo explicativo relacional de movilidad social propone una relación entre los microprocesos de movilidad, consumo y acumulación con las dinámicas macroestructurales de la sociedad del consumo, el sistema capitalista y los campos del mercado y la desigualdad.

Esta relación entre lo micro y lo macro se manifiesta en forma de paradoja, ya que el agente alimenta y fortalece el sistema y la estructura que busca superar a través de la movilidad. El capitalismo junto con el mercado establece normas de apropiación de los bienes y servicios que ofertan, mientras la cultura y la sociedad de consumo retoma estas normas, significa los bienes y configura sistemas de creencias que funcionan a la par de las leyes del mercado.

El campo de la desigualdad se fortalece tras estos procesos, tornándose legítimo y naturalizado. Así, la estructura junto con sus sistemas y campos no solo enmarcan estas dinámicas de movilidad y ganancia de estatus, sino que la provocan, alimentan y consolidan. El agente actúa en conformidad porque considera que realiza lo "propio" o "aceptable" y aunque se plantee cuestionamientos frente a lo injusto del mercado y de la desigualdad que genera, termina validando esta conflictiva relación por no encontrar otros caminos de resolución y por evaluarlas como parte de su cultura y sociedad.

Revista RBBA $\mid$ Revista Binacional Brasil Argentina Vol. 10, num. 2, dez/2021, p. 78-92 


\section{A MODO DE CONCLUSIÓN: ALCANCE DEL MODELO}

Ante a las condiciones de exclusión y desigualdad presentes en el contexto colombiano, las clases medias y bajas en búsqueda de estatus acallan sus demandas sociales y actúan de conformidad con el sistema capitalista, ofrendando su esfuerzo personal y enorgulleciéndose de los signos de éxito y prestigio que les provee el mercado.

Los consumidores estatutarios naturalizan la desigualdad porque aceptan las condiciones estructurales de desigualdad y sus situaciones de asimetría, así como los mecanismos de explotación y acaparamiento de oportunidades, básicos en la estabilización del sistema de desigualdades categoriales. Los sujetos negocian sus acciones solidarias y actúan de conformidad con los habitus de las estructuras jerárquicas impuestas por el sistema económico y de mercado, base sobre el que opera la cultura y la sociedad de consumo.

Esta dinámica de relación entre el capitalismo, el estatus y el mercado es recogida en Modelo explicativo relacional de movilidad de clases sociales que expone las dinámicas entre aspectos macro y micro y las lógicas de sus relaciones. Evidencia a partir de su explicación dos paradojas. La primera paradoja manifiesta que el plan de escape de la inequidad reproduce una nueva inequidad; y la segunda paradoja se basa en que evadir las nuevas desigualdades requiere de estrategias de acumulación de ciertos capitales y de la conformidad con los mecanismos constitutivos de la desigualdad, legitimándolos y naturalizándolos.

Estas paradojas, surgidas a partir de una mirada crítica sobre los datos, configuran una sentencia de culpabilidad (o complicidad) en el delito de generar desigualdad social de los agentes de las clases medias y bajas sin considerar otros elementos que sopesen el veredicto, como el papel de la tradición cultural de desigualdad, los requerimientos de las identidades sociales de clase y las intersubjetividades sobre el ideario del desarrollo personal que determinan los esfuerzos del sujeto en una contienda social por el estatus, por el respeto y por la posibilidad de ser y de ofrecer a su familia un fututo menos tortuoso.

Este modelo no pretende justificar los medios y mecanismos de exclusión e inequidad, sino explicar las dinámicas de estos medios para desarrollar una conciencia de clase que entre a cuestionar la intromisión del sistema económico en la vida social y en la búsqueda de un bienestar desde los lazos de la solidaridad y empatía. Además, el estudio de las pautas de

\begin{tabular}{l|l} 
Revista RBBA & Revista Binacional Brasil Argentina Vol. 10, num. 2, dez/2021, p. 78-92
\end{tabular} 
movilidad social en un contexto particular es un indicador importante para entender los procesos de cambio social y de las configuraciones que desarrolla la sociedad actual.

\section{REFERENCIAS}

AGO, R. Conspicuous consumption. En: Encyclopedia of Early Modern History online. Brill, 2015, p. 2005-2012. https://doi.org/10.1163/2352-0272_emho_com_028228

ARIAS, R. Historia de Colombia contemporánea (1920-2010). Bogotá: Ministerio de Cultura. Biblioteca Nacional de Colombia, 2017.

BAUMAN, Z. Identidad. Editorial Losada. 2007.

BHATTACHARJEE, A., BERGER, J. Y MENON, G. When Identity Marketing Backfires: Consumer Agency in Identity Expression. Journal of Consumer Research, n 41, p.294 -309, 2014. http:// 294-309. 10.1086/676125.

BECKER, G. A treatise on the family. Cambridge, Harvard University Press.1993, 198p.

BELTRÁN, W. Pluralización religiosa y cambio social en Colombia. Theologica Xaveriana, 63(175), p. 57-85, 2013. http://www.scielo.org.co/scielo.php?script=sci_arttext\&pid=S0120$36492013000100003 \& \operatorname{lng}=$ en\&tlng=es

BOURDIEU, P. Espacio social y génesis de las clases en Sociología y cultura. Editorial Grijalbo. 1990.

CAMPBELL, C. Conspicuous Confusion. A Critique of Veblen's Theory of Conspicuous Consumption. American Sociological Association, 13(1), pp.37-47, 1995. https://www.jstor.com/stable/202004

DE BOTTON. A. La ansiedad por el estatus. Madrid: Editorial Taurus, 2004.

DEATON, A. El gran escape. Salud, riqueza y los orígenes de la desigualdad. Bogotá: Fondo de Cultura Económica, 2015.

FEATHERSTONE, M. Cultura de consumo y posmodernismo. Argentina: Amorrortu, 2001

GORGA, R., LEITES, M. Y VIGORITO A. El consumo de bienes visibles y el rol del grupo de referencia. Un análisis para cuatro países latinoamericanos. Serie Documentos de Trabajo, DT 13/2016. Uruguay: Instituto de Economía, Facultad de Ciencias Económicas y Administración, Universidad de la República, 2016.

HARVEY, D. Diecisiete contradicciones y el fin del capitalismo. Quito: IAEN-Instituto de Altos Estudios Nacionales del Ecuador, 2014.

HARVEY, D. Breve historia del Neoliberalismo. Madrid: Ediciones Abtl, S. A, 2007.

Revista RBBA $\mid$ Revista Binacional Brasil Argentina Vol. 10, num. 2, dez/2021, p. 78-92 
HERNÁNDEZ SAMPIERI, R., FERNÁNDEZ COLLADO, C., Y BAPTISTA LUCIO, P. Metodología de la investigación. México D.F.: McGraw-Hill, 2008.

ILLOUZ, E. Intimidades congeladas. Las emociones en el capitalismo. Katz Editores, 2007.

KAIPPER CERATT, M. Latinoamérica, más cerca de la clase media que de la pobreza. Banco Mundial Noticias. (29 de enero de 2016). http://www.bancomundial.org/es/news/feature/2016/01/29/vulnerables-latinoamerica-mascerca-clase-media-pobreza

LEWELLEN, T. The Anthropology of globalization. Cultural Anthropology Enters the 21st Century. United States of America: Greenwood Publishing Group, Inc, 2002.

MILLER, D. Consumption and commodities. Annual Review of Anthropology, n 24, p.141$161,1995$.

OLIVEROS, D. El precio de acceder a lo digital: conflictos de centralidad entre el capital cultural y el capital TIC en clases medias y bajas de Bogotá, Colombia. Contratexto, n 28, p.93-116, 2017. https://doi.org/10.26439/contratexto2017.n028.1552

PAYNE, M. Diccionario de teoría crítica y estudios culturales. Buenos Aires: Paidós, 2002.

PEDRAZA, Z., En cuerpo y alma. Visiones del progreso y la felicidad: educación, cuerpo y orden social en Colombia (1830-1990). Bogotá. Universidad de los Andes. Ediciones Uniandes, 2011.

SCHOR, J. Conspicuous Consumption. En Thomas Cook, D. (Ed.). The Blackwell Encyclopedia of Consumption and Consumer Studies. Chicago: John Wiley y Sons, 2015.

VIVAS PACHECO, H. ¿Qué es la clase media en Colombia? Razón Pública.com. (11 de febrero de 2019). https://www.razonpublica.com/index.php/econom-y-sociedad-temas29/11753-que-es-la-clase-media-en-colombia.html

Revista RBBA $\mid$ Revista Binacional Brasil Argentina Vol. 10, num. 2, dez/2021, p. 78-92 